\title{
IgG4-Related Disease Affecting Testicle and Myelodysplastic Syndrome: Just a Coincidence?
}

\author{
Carla Alvarez Gonzalez ${ }^{1}{ }_{2}$ Juan Luis Carrillo Linares ${ }^{1}$, Isabel García Muñoz², Andrea Escalona García², Pedro Valdivielso ${ }^{1,3}$ \\ ${ }^{1}$ Department of Internal Medicine, Hospital Universitario Virgen de la Victoria, Málaga, Spain \\ ${ }^{2}$ Department of Pathological Anatomy, Hospital Universitario Virgen de la Victoria, Málaga, Spain \\ ${ }^{3}$ Faculty of medicine, University of Malaga, Malaga, Spain
}

Received: 07/10/2021

Accepted: $19 / 10 / 2021$

Published: $28 / 12 / 2021$

How to cite this article: Alvarez Gonzalez C, Carrillo Linares JL, García Muñoz I, Escalona García A, Valdivielso P. IgG4-related disease affecting testicle and myelodysplastic syndrome. EJCRIM 2021;8: doi:10.12890/2021_002981.

Conflicts of Interests: The authors declare there are no competing interests.

This article is licensed under a Commons Attribution Non-Commercial 4.0 License

\section{ABSTRACT}

Immunoglobulin G4-related disease (IgG4-RD) is progressive immune-mediated fibrotic condition characterised by a tendency to form tumefactive lesions in different organs and by elevated IgG4 serum concentrations. Urological manifestations are rare and normally occur together with other systemic affections. Myelodysplastic syndromes (MDS) are hematopoietic stem cell neoplasms, with different subtypes based on the type of blood cells involved. MDS can be associated with other pathologies or medical treatments. We present an uncommon case of testicular manifestation associated with myelodysplastic syndrome here.

\section{LEARNING POINTS}

- Testicular inflammation is a manifestation of IgG4-related disease.

- Testicular inflammation and myelodysplastic syndrome can simultaneously present.

\section{KEYWORDS}

Immunoglobulin G4-related disease, IgG4-RD, myelodysplastic syndrome, testicle

\section{INTRODUCTION}

IgG4-related disease (IgG4-RD) is a fibroinflammatory condition characterised by a tendency to form tumour-like masses. The clinical manifestations are heterogeneous and frequently of insidious onset, commonly presenting with constitutional syndrome. The histopathological features are consistent across all affected organs. They include lymphoplasmacytic infiltration with a high percentage of IgG4 plasma cells, a characteristic pattern of fibrosis, tissue eosinophilia, and obliterative phlebitis. The disease is more prevalent in adults, especially elderly males. Fever or high C-reactive protein levels are uncommon and IgG4 serum levels are typically elevated. The importance of an early diagnosis is crucial so that malignancy can be ruled out. A definitive diagnosis can only be made by biopsy and lgG4-RD is treated specifically with rituximab, which was highly successful in clinical trials ${ }^{[1]}$.

Myelodysplastic syndromes (MDS) are hematopoietic clonal stem cell disorders causing cytopenia that occasionally progress to acute myeloid leukaemia. MDS can be idiopathic or secondary to genetic disorders, radiation, cytostatic agents, malignancies, etc. However, their association with $\operatorname{lgG} 4-\mathrm{RD}$ is rare. 


\section{CASE DESCRIPTION}

We present the case of an 82-year-old man who complained of progressive malaise. He was previously diagnosed with arterial hypertension, hypercholesterolaemia, type 2 diabetes, mitral valve prolapse, and prostatic adenoma. He was being treated with hydrochlorothiazide $50 \mathrm{mg}$ daily; dutasteride/tamsulosin 0.5/0.4 mg daily; enalapril $20 \mathrm{mg}$ daily, gliclazide $30 \mathrm{mg}$ three times daily; metformin $850 \mathrm{mg}$ three times daily; omeprazole $20 \mathrm{mg}$ daily; triflusal $300 \mathrm{mg}$ twice daily; simvastatin $10 \mathrm{mg}$ daily; and verapamil $80 \mathrm{mg}$ twice daily. Six months prior to admission, he was referred to our hospital with progressive general deterioration, asthenia, hyporexia with unexplained weight loss, postprandial nausea, and constipation with normal stool characteristics. Additionally, he complained of right testicular enlargement, followed by enlargement of the left testicle with postural discomfort. A clinical examination revealed pallor, a reducible umbilical hernia, modest enlargement of both testicles, with non-inflammatory signs and petechial exanthema on his lower limbs.

Methods and procedures

Laboratory investigations were conducted and analytical data are shown in Table 1.

\begin{tabular}{|c|c|c|}
\hline Parameter & Patient data & Normal range \\
\hline Haemoglobin (g/dl) & 8.1 & $13-17.5$ \\
\hline$M C V(f l)$ & 98.8 & $80-100$ \\
\hline Platelets $\left(\times 10^{9} / \mathrm{L}\right)$ & 8 & $140-450$ \\
\hline Leukocytes (×109/L) & 12 & $4.4-11.3$ \\
\hline Neutrophils $\left(\times 10^{9} / \mathrm{L}\right)$ & 9.4 & $1.8-7.7$ \\
\hline Ferritin (ng/mL) & 2325 & $22-322$ \\
\hline$L D H(U / L)$ & 282 & $120-246$ \\
\hline Beta 2 microglobulin $(\mathrm{mg} / \mathrm{L})$ & 6.46 & $0.7-1.8$ \\
\hline Immunoglobulin (Ig) A (mg/dL) & 596 & $40-350$ \\
\hline $\lg G(m g / d L)$ & 2103 & $650-1600$ \\
\hline $\lg G 1(\mathrm{mg} / \mathrm{dL})$ & 1320 & $278-822$ \\
\hline $\lg G 4(\mathrm{mg} / \mathrm{dL})$ & 321 & $3-132$ \\
\hline C-reactive protein $(C R P)(\mathrm{mg} / \mathrm{L})$ & 66 & $<5$ \\
\hline Albumin (g/dL) & 3 & $3.4-4.8$ \\
\hline Gamma globulin (g/dL) & 1.6 & $0.8-1.4$ \\
\hline Albumin/globulin (A/G) ratio & 0.8 & $1.2-2.2$ \\
\hline Kappa light chain (mg/L) & 146 & $3.3-19.4$ \\
\hline Lambda light chain (mg/L) & 83.4 & $5.7-26.3$ \\
\hline Free kappa/free lambda ratio & 1.75 & $0.26-1.65$ \\
\hline
\end{tabular}

Table 1. Key analytical data

A monoclonal component was not found and the immunofixation electrophoresis was negative. Antinuclear antibodies and anti-dsDNA serology to HIV, hepatitis C virus, hepatitis B virus, Epstein-Barr virus, cytomegalovirus, and blood and urine cultures were all negative. Urinalysis only revealed the presence of proteinuria $(533 \mathrm{mg} / 24 \mathrm{~h})(<80)$, with no detection of light chain immunoglobulins. A peripheral blood smear showed a leukoerythroblastic reaction in isolated immature cells with high nuclear-cytoplasmic ratio and lax chromatin. 
Thoracic X-ray, colonoscopy, and gastroscopy findings were unremarkable. A testicular ultrasound showed normal size and morphology of the testes, presenting a bilateral striated hypoechoic pattern more remarkable in the right side, with normal and symmetric vascularisation. There was a thickening of the epididymis and the spermatic cord with increased echogenicity of both sides. There was a large hyperechogenic extratesticular collection, with septa and liquid levels in the interior. These findings suggested an evolved hematocele. (Fig. 1). Two nonspecific hypermetabolic foci were found on a PET scan in both testicles.
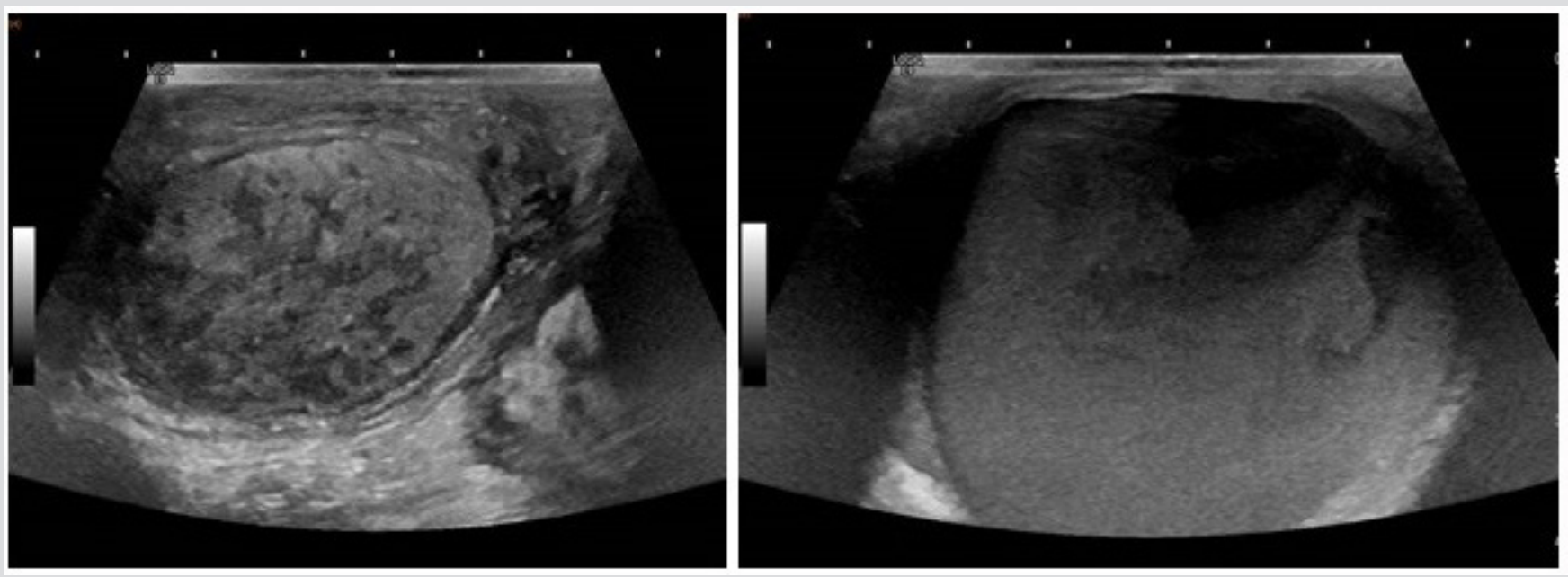

Figure 1. Testicular ultrasound showing a parenchymatous striated hypoechoic pattern (left) and a large collection suggestive of a hematocele (right)

A bone marrow aspiration revealed hypercellularity with dysgranulopoiesis and excess blast cells (9\%). There were also abnormalities in the immunophenotype of granulocytic, monocyte, and erythroid lineages.

Arightorchidectomy was performed dueto the high suspicion of malignancy. The histological examination revealed diffuse lymphoplasmacytic infiltration affecting the tubules, rete testis, and epididymis. It showed a polytypic light chain expression (kappa>lambda) and a high immunoexpression of IgG4 (Fig. 2).
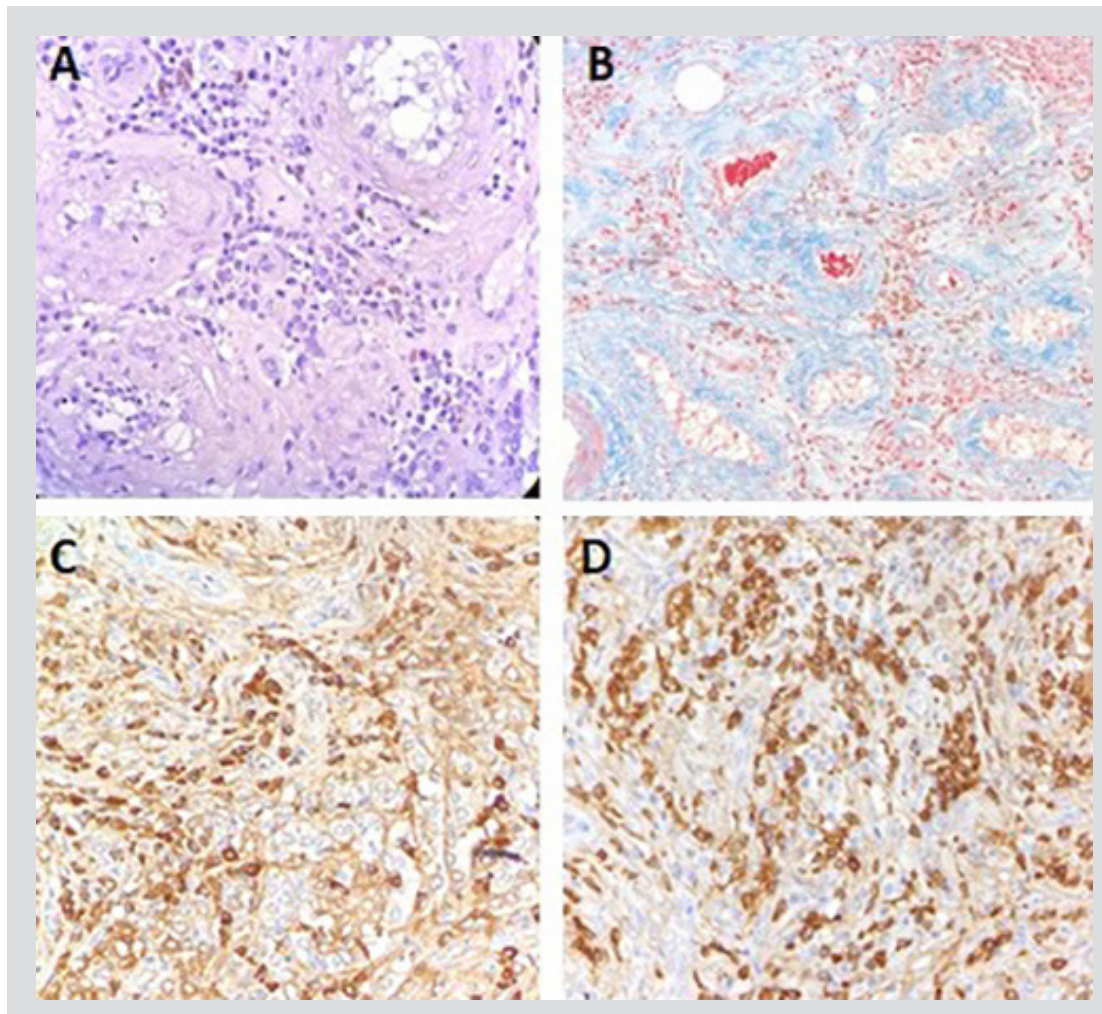

Figure 2. Right orchidectomy (pathological anatomy) showing diffuse lymphoplasmacytic infiltration (A), with peritubular fibrosis in Mallory's trichrome staining (B), positive lgG staining in immunohistochemistry (C), with a percentage of lgG4 more than $40 \%$ (D). 
The patient was diagnosed with IgG4 testicular disease and MDS with excess blasts type 1 (WHO Classification 2016). It was decided to start conservative treatment with blood transfusions for MDS and a short regimen of corticosteroids for IgG4-RD. The outcome was unfavourable due to the advanced stage of the haematological disease, resulting in the death of the patient only a few months after diagnosis.

\section{DISCUSSION}

Urological IgG4-RD is rare and normally associated with other organic manifestations. Few cases have been reported involving the testes. There is one case report of a bilateral epididymo-orchitis associated with a pancreatic presentation ${ }^{[2]}$. Another publication describes a painless unilateral hydrocele with prostatic, pancreatic, and retroperitoneal manifestations ${ }^{[3]}$. There is only one report of a patient with an exclusive testicular manifestation of the disease as a pseudotumour ${ }^{[4]}$. All these cases were diagnosed by histological examination in order to rule out malignancy.

Given the heterogeneous presentation and the tendency to simulate other pathologies, we should consider introducing lgG4-RD in the differential diagnoses of multiple diseases. This includes multisystemic and mass-like syndromes independent of location. Even though IgG4-RD has been more frequently described in other organs, it should always be taken into consideration.

There are two case reports of a simultaneous diagnosis of MDS and IgG4-RD. The first one developed as an autoimmune pancreatitis with submandibular and lachrymal gland infiltration ${ }^{[5]}$, while the second case was an encephalopathy with a possible diagnosis of ER-IgG4 associated with MDS, although Castleman disease could not be definitively ruled out ${ }^{[6]}$.

There is strong evidence of the association between MDS and different systemic autoimmune conditions. Although the precise underlying mechanism remains unclear, there is growing evidence for the role of activated immunity and immune deregulation in the pathogenesis of MDS. Therefore, we suggest a correlation between the development of these disorders, being both diagnoses more than a simple coincidence.

\section{CONCLUSION}

We describe the case of a rare presentation of IgG4-RD occurring with an MDS diagnosis. This highlights the variability of the presentation of IgG4-RD and shows an association between two pathologies that should not go undetected.

\section{REFERENCES}

Perugino CA, Stone JH. IgG4-related disease: an update on pathophysiology and implications for clinical care. Nat Rev Rheumatol 2020;16(12):702-714. de Buy Wenniger LM, Scheltema JM, Verheij J, Beuers U. Testicular inflammation as a new manifestation of IgG4-associated disease. Urology 2013;82(2):e15-16.

Tokura Y, Kobayashi M, Kusakabe T, Kamai T. Case of hydrocele testis caused by vaginalitis, a rare manifestation of immunoglobulin G4-related disease. Int $J$ Urol 2017;24(10):771-773.

4. Lal J, Bhat S, Doddamani S, Devi L. Isolated testicular immunoglobulin G4-related disease: a mimicker of malignancy. Indian J Urol 2016;32(4):326-328.

5. Tabata R, Tabata C, Okamoto T, Omori K, Terada M, Nagai T. Autoimmune pancreatitis associated with myelodysplastic syndrome. Int Arch Allergy Immunol 2010;151(2):168172.

6. Matsumoto N, Hishikawa N, Ikegami K, Sato K, Omote Y, Takemoto M, et al. A unique case of encephalopathy with an elevated IgG-4 and extremely high interleukin-6 level and delayed myelodysplastic syndrome. Intern Med 2021;60(13):2125-2128. 